\title{
ON THE KARLIN-MCGREGOR THEOREM AND APPLICATIONS
}

\author{
By W. BÖHM AND S. G. MOHANTY
}

\author{
University of Economics and McMaster University
}

\begin{abstract}
In this paper we present some interesting results which follow from the celebrated determinant formulas for noncoincidence probabilities of Markov processes discovered by Karlin and McGregor. The first theorem is a determinant formula for the probability that a Markov jump process will avoid a certain finite set of points. From this theorem a simple solution of the moving boundary problem for certain types of Markov processes can be obtained. The other theorems deal with noncoincidence probabilities of sets of random walks which need not be identically distributed. These formulas have interesting applications, especially in the theory of queues.
\end{abstract}

1. Introduction. In their study of the Stieltjes moment problem and birth-death processes, Karlin and McGregor (1957) observed that determinants formed by the transition functions $P_{i j}(t)$ of birth-death processes are strictly positive for $t>0$. In a subsequent paper [Karlin and McGregor (1959)] this observation was given the following interesting probabilistic interpretation.

Consider a strong Markov process whose state space is a metric space and whose sample paths are continuous on the right. Select $n$ states $x_{1}, x_{2}, \ldots, x_{n}$ and $n$ Borel sets $E_{1}, E_{2}, \ldots, E_{n}$ and form the determinant

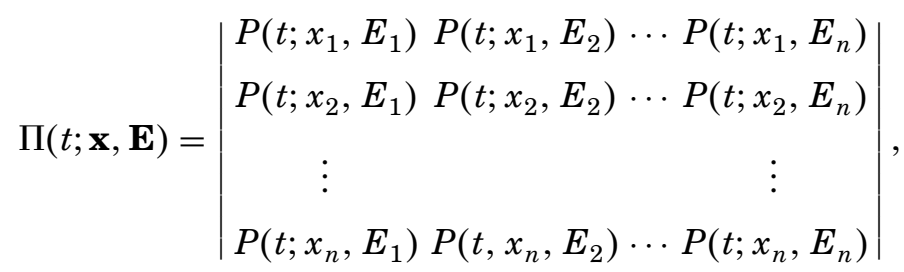

where $\mathbf{x}=\left(x_{1}, x_{2}, \ldots, x_{n}\right), \mathbf{E}=\left(E_{1}, E_{2}, \ldots, E_{n}\right)$ and $P\left(t ; x_{i}, E_{j}\right)$ denotes the transition function of the process. Suppose that $n$ labeled particles start in states $x_{1}, \ldots, x_{n}$ and execute the process simultaneously and independently. For each permutation $\sigma$ of $1,2, \ldots, n$ let $A_{\sigma}$ denote the event that at time $t$ the particles are in the states $E_{\sigma_{1}}, \ldots, E_{\sigma_{n}}$ without any two of them having ever been coincident in the intervening time, coincidence meaning that at least two particles occupy the same state at the same time. Then

$$
\Pi(t ; \mathbf{x}, \mathbf{E})=\sum_{\sigma}(\operatorname{sign} \sigma) P\left(A_{\sigma}\right),
$$

where the summation runs over all permutations $\sigma$.

Received May 1996; revised November 1996.

AMS 1991 subject classifications. 60C05, 60J15.

Key words and phrases. Noncoincidence probabilities, moving boundaries, order statistics, queueing. 
The above result was generalized by Karlin (1988) to sets of nonstationary Markov processes with arbitrary starting and stopping times.

Consider $n$ independent Markov processes $X_{1}(t), X_{2}(t), \ldots, X_{n}(t)$, not necessarily stationary, governed by the same transition function

$$
P(s, x ; t, E)=P(X(t) \in E \mid X(s)=x) .
$$

Let $\mathbf{X}=\left(X_{1}, \ldots, X_{n}\right)$ and define $\mathbf{X}(\mathbf{u})=\left(X_{1}\left(u_{1}\right), \ldots, X_{n}\left(u_{n}\right)\right)$. Now suppose that the starting times of $\mathbf{X}$ are given by $\mathbf{s}=\left(s_{1}, \ldots, s_{n}\right)$ and assume that the processes start in positions $\alpha_{1}, \ldots, \alpha_{n}$. Thus $\mathbf{X}(\mathbf{s})=\boldsymbol{\alpha}$. Furthermore assume the processes stop at times $\mathbf{t}=\left(t_{1}, \ldots, t_{n}\right)$ in positions $\boldsymbol{\beta}=\left(\beta_{1}, \ldots, \beta_{n}\right)$, with $s_{i} \leq t_{i}$, so $\mathbf{X}(\mathbf{t})=\boldsymbol{\beta}$. The transition of the $i$ th process from state $\alpha_{i}$ to state $\beta_{i}$ during the time interval $\left(s_{i}, t_{i}\right), i=1,2, \ldots, n$, will be denoted by

$$
[\mathbf{X}, \mathbf{s}, \boldsymbol{\alpha}] \rightarrow[\mathbf{X}, \mathbf{t}, \boldsymbol{\beta}] .
$$

Karlin has proved the following theorem.

THEOREM 1.1 [Karlin (1988)]. Let $\sigma$ represent a permutation on $(1,2, \ldots$, $n)$ and let $\boldsymbol{\beta}_{\sigma}=\left(\beta_{\sigma(1)}, \beta_{\sigma(2)}, \ldots, \beta_{\sigma(n)}\right)$ and $\mathbf{t}_{\sigma}=\left(t_{\sigma(1)}, t_{\sigma(2)}, \ldots, t_{\sigma(n)}\right)$. Then

$$
\begin{aligned}
& \sum_{\sigma}(\operatorname{sign} \sigma) P\left([\mathbf{X}, \mathbf{s}, \boldsymbol{\alpha}] \rightarrow\left[\mathbf{X}, \mathbf{t}_{\sigma}, \boldsymbol{\beta}_{\sigma}\right]\right) \\
& \quad=\sum_{\sigma}(\operatorname{sign} \sigma) P\left([\mathbf{X}, \mathbf{s}, \boldsymbol{\alpha}] \rightarrow\left[\mathbf{X}, \mathbf{t}_{\sigma}, \boldsymbol{\beta}_{\sigma}\right] \text { without coincidence }\right) .
\end{aligned}
$$

As it stands, (3) seems to be of limited value only. However, many remarkable results follow from (3), if the only permutation where no coincidence occurs is the identity, in which case on the right-hand side of (3) only one term survives. This has been shown by Karlin and McGregor (1959) and Karlin (1988). Further examples of this interesting special case are given below.

An analogue of Theorem 1.1 has been discovered by Gessel and Viennot (1985) in lattice path combinatorics, where a determinant formula [essentially equal to (3)] is used to count sets of nonintersecting two-dimensional lattice paths. Karlin (1988) points out that several nontrivial combinatorial theorems may be obtained from (3) directly. Particular examples are the $n$ candidate ballot problem, monotone dominance orderings and counting sets of nonintersecting paths. There are many other interesting applications in various fields, notably in statistical mechanics, where this result is used, for instance, in the study of phase transitions of almost one-dimensional systems [Fisher (1984)].

In this paper we will present some more results which follow from this theorem. The first result provides a simple solution of the moving boundary problem for certain types of Markov processes, a problem of particular importance, for instance, in sequential statistics. As a reference on moving boundaries and sequential statistics, see Siegmund (1985). The other theorems are an extension of the Karlin-McGregor theorem to sets of nonidentical or dissimilar Markov processes. If the processes have different transition functions, then 
in general no simple determinant formulas [comparable to (3)] can be given. However, for simple random walks and Poisson counting processes noncoincidence probabilities can be expressed as determinants, and it turns out that some interesting problems in the theory of queues may be solved in this way.

2. The theorems. The first theorem provides a useful result for Markov jump processes which are not allowed to enter certain discrete point sets.

THEOREM 2.1. Let $X_{t}$ be a strong Markov process with discrete state space $\mathscr{S} \subset \mathbb{Z}$ and transition function

$$
P\left(X_{t}=k \mid X_{s}=m\right)=p(s, t ; m, k), \quad s \leq t .
$$

Furthermore, let

$$
\mathscr{C}_{M}=\left\{\left(u_{1}, a_{1}\right),\left(u_{2}, a_{2}\right), \ldots,\left(u_{M}, a_{M}\right)\right\}, \quad u_{i} \in \mathbb{R}^{+}, a_{i} \in \mathscr{P}
$$

denote a set of points ordered such that $u_{1} \leq u_{2} \leq \cdots \leq u_{M}$. The first coordinate of these points represents time, the second a possible state of the process $X_{t}$.

Then the probability that $X_{t}$ moves from state $m$ to state $k$ in $(0, t)$ without touching any of the points in $\mathscr{C}_{M}$ is given by the determinant

$$
P\left(X_{t}=k, X_{s} \notin \mathscr{C}_{M}, 0 \leq s \leq t \mid X_{0}=m\right)=\operatorname{det}\left\|d_{i j}\right\|_{(M+1) \times(M+1)},
$$

where

$$
\begin{array}{rlrl}
d_{00}= & p(0, t ; m, k) & \\
d_{0 j}= & p\left(u_{j}, t ; a_{j}, k\right), & & j=1,2, \ldots, M \\
d_{i 0}= & p\left(0, u_{i} ; m, a_{i}\right), & i=1,2, \ldots, M \\
d_{i j}= & p\left(u_{j}, u_{i} ; a_{j}, a_{i}\right), & i \geq j \\
& 0 \quad \text { otherwise, } & &
\end{array}
$$

and $X_{t} \notin \mathscr{C}_{M}$ is a shorthand notation for $\left(t, X_{t}\right) \notin \mathscr{C}_{M}$.

This determinant may be evaluated recursively. In particular:

$$
P\left(X_{t}=k, X_{s} \notin \mathscr{C}_{M}, 0 \leq s \leq t \mid X_{0}=m\right)=P_{M+1},
$$

where

$$
P_{\ell}=-\sum_{i=0}^{\ell-1} w_{\ell i} P_{i} \quad \text { for } \ell=1,2, \ldots, M+1
$$

with $P_{0}=-1$ and

$$
w_{i j}= \begin{cases}p\left(u_{j}, u_{i} ; a_{j}, a_{i}\right), & 0 \leq i, j \leq M+1, \\ 0, & \text { if } u_{j}>u_{i},\end{cases}
$$


and boundary conditions

$$
u_{0}=0, \quad a_{0}=m, \quad u_{M+1}=t, \quad a_{M+1}=k .
$$

Proof. Consider $M+1$ copies of the Markov process $X_{t}$, say $X_{t}^{0}, X_{t}^{1}, \ldots$, $X_{t}^{M}$. Suppose now that $X_{t}^{0}$ starts at $(0, m)$ and terminates at $(t, k)$. The other processes $X_{t}^{i}, i=1, \ldots, M$ start and stop in $\left(u_{i}, a_{i}\right)$; thus their sample paths have length zero. Now apply Theorem 1.1 to this set of paths. It is clear that the only permutation of the terminal positions, where no coincidence occurs, is the identity permutation. To see this, it is sufficient to consider $X_{t}^{0}$ and $X_{t}^{i}$, for some $i>0$. If we interchange the terminal points of these two processes, then $X_{t}^{0}$ must move from $(0, m)$ to $\left(u_{i}, a_{i}\right)$ and $X_{t}^{1}$ from $\left(u_{i}, a_{i}\right)$ to $(t, k)$. Hence a coincidence necessarily occurs in the point $\left(u_{i}, a_{i}\right)$. Thus the contributions to the right-hand side of (3) for all permutations other than the identity are zero and the result follows. The recurrence relation (7) is easily obtained by interchanging the first and the last row in the determinant (4) and expanding this determinant according to the expansion theorem of Laplace.

The idea of this proof has been borrowed from Stanley [(1986), page 84]. Krattenthaler and Mohanty (1992) have already shown the power of this approach in counting Young tableaux.

Next we generalize the Karlin-McGregor theorem to dissimilar processes, that is, processes which have different transition functions. If the processes are binomial random walks or counting processes of Bernoulli or Poisson type, then noncoincidence probabilities can be given in form of determinants quite similar to (3). Unfortunately, only in exceptional cases can formulas similar to (3) be found. The difficulties we encounter here are due to the fact that interchanging sample paths segments of the processes after the point of first coincidence (it is this idea on which the proof of Theorem 1.1 is based) will change the probability distribution of the paths.

Let us discuss the case of dissimilar binomial random walks first.

THEOREM 2.2. Let $X_{1}, \ldots, X_{r}$ denote a set of independent binomial random walks, where $X_{i}$ has jumps of magnitude +1 and -1 with probabilities $p_{i}$ and $q_{i}=1-p_{i}$. Assume that the random walks start at time zero in positions $\alpha_{1}<\alpha_{2}<\cdots<\alpha_{r}$ and terminate at time $n$ in positions $\beta_{1}<\beta_{2}<\cdots<\beta_{r}$, the $\alpha_{i}$ being all either odd or even.

Then the only permutation $\sigma$ of the terminal positions $\beta_{i}$ where no coincidence of the random walks $X_{i}$ occurs is the identity and

$$
\begin{aligned}
& P([\mathbf{X}, 0, \boldsymbol{\alpha}] \rightarrow[\mathbf{X}, n, \boldsymbol{\beta}] \text { without coincidence }) \\
&=\operatorname{det}\left\|\left(\begin{array}{c}
n \\
\left(n+\beta_{i}-\alpha_{j}\right) / 2
\end{array}\right)\right\| \prod_{\nu=1}^{r} p_{\nu}^{\left(n+\beta_{\nu}-\alpha_{\nu}\right) / 2} q_{\nu}^{\left(n-\beta_{\nu}+\alpha_{\nu}\right) / 2} .
\end{aligned}
$$

PROOF. Assume first that the random walks $X_{i}$ are identically distributed and symmetric so that for each $X_{i}$, positive and negative jumps have prob- 
ability $\frac{1}{2}$. Then any permutation $\sigma$ other than $\sigma=i d$ will necessarily entail that at least two of the random walks $X_{i}$ will have a coincidence.

Thus on the right-hand side of (3), only the term corresponding to $\sigma=i d$ will survive and it follows that

$$
P([\mathbf{X}, 0, \boldsymbol{\alpha}] \rightarrow[\mathbf{X}, n, \boldsymbol{\beta}], \text { without coincidence })
$$

$$
=\operatorname{det}\left\|\left(\begin{array}{c}
n \\
\left(n+\beta_{i}-\alpha_{j}\right) / 2
\end{array}\right)\right\|_{r \times r} 2^{-n r} .
$$

Since any sample path of $X_{i}$ has probability $2^{-n}$, the determinant on the right-hand side of (9) equals the number of sets of sample paths such that $[\mathbf{X}, 0, \boldsymbol{\alpha}] \rightarrow[\mathbf{X}, n, \boldsymbol{\beta}]$ without coincidence.

Now assume that process $X_{i}$ has jumps of magnitude +1 and -1 with probabilities $p_{i}$ and $q_{i}$, respectively. The probability of a sample path is in this case

$$
p_{i}^{\left(n+\beta_{i}-\alpha_{i}\right) / 2} q_{i}^{\left(n-\beta_{i}+\alpha_{i}\right) / 2},
$$

Multiplying the determinant in (9) by these probabilities yields (8).

A result equivalent to Theorem 2.2 is the following: let $X_{1}, \ldots, X_{r}$ denote a set of independent Bernoulli processes, where $X_{i}$ has jumps of magnitude +1 and 0 with probabilities $p_{i}$ and $q_{i}=1-p_{i}$. Assume that the processes start at time zero in positions $\alpha_{1}<\alpha_{2}<\cdots<\alpha_{r}$ and terminate at time $n$ in positions $\beta_{1}<\beta_{2}<\cdots<\beta_{r}$.

Then again the only permutation $\sigma$ of the terminal positions $\beta_{i}$ where no coincidence of the processes $X_{i}$ occurs is the identity and

$$
\begin{gathered}
P([\mathbf{X}, 0, \boldsymbol{\alpha}] \rightarrow[\mathbf{X}, n, \boldsymbol{\beta}] \text { without coincidence }) \\
=\operatorname{det}\left\|\left(\begin{array}{c}
n \\
\beta_{i}-\alpha_{j}
\end{array}\right)\right\| \prod_{\nu=1}^{r} p_{\nu}^{\beta_{\nu}-\alpha_{\nu}} q_{\nu}^{n-\beta_{\nu}+\alpha_{\nu}} .
\end{gathered}
$$

The next case we consider is of particular importance in queueing applications.

TheOREM 2.3. Let $X_{1}, \ldots, X_{r}$ denote $r>1$ independent Poisson counting processes with rates $\lambda_{1}, \ldots, \lambda_{r}$ and transition probabilities

$$
P\left(X_{i}(t)=k \mid X_{i}(0)=m\right)=\frac{\exp \left(-\lambda_{i} t\right)\left(\lambda_{i} t\right)^{k-m}}{(k-m) !} .
$$

Assume that the processes $X_{i}$ start at time zero in positions $m_{i}$ with $m_{1}<$ $m_{2}<\cdots<m_{r}$ and terminate at time $t$ in positions $k_{i}, k_{1}<k_{2}<\cdots<k_{r}$. Let 
$\mathbf{m}=\left(m_{1}, \ldots, m_{r}\right)$ and $\mathbf{k}=\left(k_{1}, \ldots, k_{r}\right)$. Then

$$
\begin{aligned}
& P([\mathbf{X}, 0, \mathbf{m}] \rightarrow[\mathbf{X}, t, \mathbf{k}] \text { without coincidence }) \\
& \quad=\operatorname{det}\left\|\frac{1}{\left(k_{i}-m_{j}\right) !}\right\| \prod_{\nu=1}^{r}\left(k_{\nu}-m_{\nu}\right) ! P([\mathbf{X}, 0, \mathbf{m}] \rightarrow[\mathbf{X}, t, \mathbf{k}]) \\
& \quad=\operatorname{det}\left\|\frac{1}{\left(k_{i}-m_{j}\right) !}\right\| \exp \left(-t \sum_{\nu=1}^{r} \lambda_{\nu}\right) \prod_{\nu=1}^{r}\left(\lambda_{\nu} t\right)^{k_{\nu}-m_{\nu}} .
\end{aligned}
$$

PRoof. This result may be proved by a limiting argument applied to (10). However, the following proof seems to be more elegant. (The authors are indebted to an anonymous referee for drawing their attention to this idea.)

Let us denote by $P_{\lambda_{1}, \ldots, \lambda_{r}}(A)$ the probability of the event $A$ when the processes $X_{i}(t), 1 \leq i \leq r$ are independent Poisson with rates $\lambda_{i}$. Given $X_{i}(0)=$ $m_{i}$ and $X_{i}(t)=k_{i}$, the $k_{i}-m_{i}$ points where $X_{i}(t)$ has jumps are uniformly distributed in $(0, t)$. Hence it follows that

$$
\begin{aligned}
& P_{\lambda_{1}, \ldots, \lambda_{r}}(\text { no coincidence } \mid \mathbf{X}(0)=\mathbf{m}, \mathbf{X}(t)=\mathbf{k}) \\
& \quad=P_{1, \ldots, 1}(\text { no coincidence } \mid \mathbf{X}(0)=\mathbf{m}, \mathbf{X}(t)=\mathbf{k}) .
\end{aligned}
$$

The right-hand side of (12) can be evaluated using the Karlin-McGregor theorem. In particular we find that

$$
\begin{aligned}
P_{1, \ldots, 1}(\text { no coincidence } \mid \mathbf{X}(0)=\mathbf{m}, \mathbf{X}(t)=\mathbf{k}) \\
=\frac{P_{1, \ldots, 1}([\mathbf{X}, 0, \mathbf{m}] \rightarrow[\mathbf{X}, t, \mathbf{k}] \text { without coincidence })}{P_{1, \ldots, 1}([\mathbf{X}, 0, \mathbf{m}] \rightarrow[\mathbf{X}, t, \mathbf{k}])} \\
=\frac{\prod_{\nu=1}^{r}\left(k_{\nu}-m_{\nu}\right) !}{e^{-r t} \prod_{\nu=1}^{r} t^{k_{\nu}-m_{\nu}}} \operatorname{det}\left\|\frac{\exp (-t) t^{k_{i}-m_{j}}}{\left(k_{i}-m_{j}\right) !}\right\| \\
=\operatorname{det}\left\|\frac{1}{\left(k_{i}-m_{j}\right) !}\right\| \prod_{\nu=1}^{r}\left(k_{\nu}-m_{\nu}\right) ! .
\end{aligned}
$$

Multiplying (13) by

$$
P([\mathbf{X}, 0, \mathbf{m}] \rightarrow[\mathbf{X}, t, \mathbf{k}])=P_{\lambda_{1}, \ldots, \lambda_{r}}([\mathbf{X}, 0, \mathbf{m}] \rightarrow[\mathbf{X}, t, \mathbf{k}])
$$

we get (11).

\section{Applications and examples.}

3.1. Birth processes with moving boundaries. Theorem 2.1 provides an efficient tool to calculate the transition functions of a large class of random walk processes which avoid certain time dependent boundaries. Of special interest is the case of pure Markov birth processes in continuous time. It is intuitively clear that a finite set of points cannot, in general, constitute an impenetrable boundary for a continuous time random walk process. However, pure birth 
processes are a notable exception, which has several interesting applications in order statistics and queueing theory.

Let $N(t), t \geq 0$ be a pure Markov birth process with jump intensities $\lambda_{i k}(t)$, $k>i \geq 0$ :

$$
P(N(t+\Delta)=k \mid N(t)=i)=\lambda_{i k}(t) \Delta+o(\Delta), \quad \Delta \rightarrow 0 .
$$

Observe that we allow $N(t)$ to be inhomogeneous in time and in space.

Now let $\beta(t)$ be a continuous and increasing function with $\beta(0) \geq 0$, which should serve as an upper boundary to $N(t)$. Crossings of this boundary from below may, of course, occur at any time and at any height. However, if the position of $N(t)$ is fixed to some integer $n<\beta(t)$, then any excursion above the boundary must be terminated by a down crossing, and such a down crossing can occur only at integer heights $0,1, \ldots, n$, since the sample paths of $N(t)$ are nondecreasing. Thus the finite set

$$
\mathscr{B}_{M}=\left\{\left(u_{i}, b_{i}\right), 1 \leq i \leq M\right\},
$$

with

$$
\begin{aligned}
b_{i} & =\lfloor\beta(0)\rfloor+i \\
u_{i} & =\beta^{-1}\left(b_{i}\right), \quad i=1,2, \ldots, n-\lfloor\beta(0)\rfloor
\end{aligned}
$$

may be used as a representation of the boundary $\beta(t)$ with $M=n-\lfloor\beta(0)\rfloor$ points, and by Theorem 2.1 and the recurrence relation (7) we obtain for $n<$ $\beta(t)$ :

$$
P(N(t)=n, N(s)<\beta(s), 0 \leq s \leq t \mid N(0)=0)=P_{M+1},
$$

with $P_{0}=-1$,

$$
P_{\ell}=-\sum_{i=0}^{\ell-1} P\left(N\left(u_{\ell}\right)=b_{\ell} \mid N\left(u_{i}\right)=b_{i}\right) P_{i}, \quad \ell=1,2, \ldots, M+1,
$$

and boundary conditions

$$
u_{0}=b_{0}=0, \quad u_{M+1}=t, \quad b_{M+1}=n .
$$

The case of a lower boundary or the two-boundary case may be handled similarly.

A particularly simple situation arises as a special case of the above result, when $N(t)$ is stationary Poisson with rate $\lambda>0$. This case has been treated earlier by different methods by Zacks (1991) and Stadje (1993).

Let

$$
\mathscr{A}_{M}=\left\{\left(u_{i}, b_{i}\right), 1 \leq i \leq M\right\}
$$

be an arbitrary boundary set ordered such that $u_{1} \leq u_{2} \leq \cdots \leq u_{M}$, and define $u_{0}=b_{0}=0, u_{M+1}=t$ and $b_{M+1}=n$, where the point $(t, n)$ does not belong to $\mathscr{A}_{M}$. Thus $\mathscr{A}_{M}$ may be an upper or lower boundary or both. Consider now the conditional probability

$$
P\left(N(s) \notin \mathscr{A}_{M}, 0 \leq s \leq t \mid N(t)=n\right),
$$


that is, the probability that $N(t)$ does not touch any of the points in $\mathscr{A}_{M}$ under the condition $N(t)=n$.

Let

$$
R_{i}=P_{i} / P\left(N\left(u_{i}\right)=b_{i}\right), \quad i=0,1, \ldots
$$

Then from the recurrence (17), it follows after dividing by $P\left(N\left(u_{\ell}\right)=b_{\ell}\right)$ that $R_{\ell}=-\sum_{i=0}^{\ell-1} \frac{P\left(N\left(u_{\ell}\right)=b_{\ell} \mid N\left(u_{i}\right)=b_{i}\right)}{P\left(N\left(u_{\ell}\right)=b_{\ell}\right)} P\left(N\left(u_{i}\right)=b_{i}\right) R_{i}, \quad i=1,2, \ldots, M+1$.

And since

$$
P\left(N\left(u_{i}\right)=b_{i}\right)=\frac{\exp \left(-\lambda u_{i}\right)\left(\lambda u_{i}\right)^{b_{i}}}{b_{i} !}
$$

we obtain the following simple and interesting recurrence satisfied by $R_{i}$ :

$$
R_{\ell}=-\sum_{i=0}^{\ell-1}\left(\begin{array}{l}
b_{\ell} \\
b_{i}
\end{array}\right)\left(1-\frac{u_{i}}{u_{\ell}}\right)^{b_{\ell}-b_{i}}\left(\frac{u_{i}}{u_{\ell}}\right)^{b_{i}} R_{i}, \quad \ell=1,2, \ldots, M+1,
$$

with boundary condition $R_{0}=-1$.

Thus

$$
P\left(N(s) \notin \mathscr{A}_{M}, 0 \leq s \leq t \mid N(t)=n\right)=R_{M+1} .
$$

The recurrence (18) has also application in order statistics.

If $T_{1} \leq T_{2} \leq \cdots \leq T_{n}$ denote the successive jump times of a stationary Poisson process, then the conditional distribution of $0 \leq T_{1} \leq T_{2} \leq \cdots \leq$ $T_{n} \leq 1$ given $N(1)=n$ is the same as the distribution of the order statistics $0 \leq \xi_{n: 1} \leq \xi_{n: 2} \leq \cdots \leq \xi_{n: n}<1$ of a sample of size $n$ drawn from the uniform $(0,1)$-distribution.

Now let $X_{1}, \ldots, X_{n}$ be i.i.d. random variables with arbitrary continuous distribution $F$ and consider

$$
Q_{n}=P\left(a_{i}<X_{n: i}<b_{i}, 1 \leq i \leq n\right),
$$

where $a_{i}, b_{i}$ are real and satisfy

$$
\begin{aligned}
& a_{1} \leq a_{2} \leq \cdots \leq a_{n}, \quad b_{1} \leq b_{2} \leq \cdots \leq b_{n}, \\
& a_{i}<b_{i}, \quad 1 \leq i \leq n .
\end{aligned}
$$

There are several examples of parameterizations of these sequences $a_{i}$ and $b_{i}$, which are important in statistical testing. The interested reader is referred to Shorack and Wellner [(1986), page 357).

Consider now (20). By the probability integral transform it follows that $F\left(X_{i}\right)$ has a uniform $(0,1)$-distribution. Thus the discussion of $(20)$ may be confined to the uniform distribution. Hence it is convenient to define real numbers $v_{i}$ and $u_{i}$ by

$$
v_{i}=F\left(b_{i}\right), \quad u_{i}=F\left(a_{i}\right), \quad i=1,2, \ldots, n .
$$


Next we form boundary sets

$$
\mathscr{A}_{n}=\left\{\left(v_{i}, i-1\right), 1 \leq i \leq n\right\} \quad \text { and } \quad \mathscr{B}_{n}=\left\{\left(u_{i}, i\right), 1 \leq i \leq n\right\}
$$

and their union

$$
\mathscr{C}_{2 n}=\left\{\left(w_{i}, c_{i}\right), 1 \leq i \leq 2 n\right\}=\mathscr{A}_{n} \cup \mathscr{B}_{n} .
$$

As usual we assume that the points in $\mathscr{C}_{2 n}$ are arranged such that $w_{1} \leq w_{2} \leq$ $\cdots \leq w_{2 n}$.

If $N(t)$ is a stationary Poisson process with rate $\lambda>0$, then by (18) we obtain the following recursion for $Q_{n}$ :

$$
\begin{aligned}
Q_{n} & =P\left(N(s) \notin \mathscr{C}_{2 n}, 0 \leq s \leq 1 \mid N(1)=n\right) \\
& =R_{2 n+1},
\end{aligned}
$$

with $R_{0}=-1$,

$$
R_{\ell}=-\sum_{i=0}^{\ell-1}\left(\begin{array}{c}
c_{\ell} \\
c_{i}
\end{array}\right)\left(1-\frac{w_{i}}{w_{\ell}}\right)^{c_{\ell}-c_{i}}\left(\frac{w_{i}}{w_{\ell}}\right)^{c_{i}} R_{i}, \quad \ell=1,2, \ldots, 2 n+1
$$

and

$$
w_{0}=c_{0}=0, \quad w_{2 n+1}=1, \quad c_{2 n+1}=n .
$$

This formula provides an interesting alternative to Noe's recurrence relation [see Shorack and Wellner (1986), pages 362) and Noe (1972)].

3.2. Applications in queueing theory. An application of Theorem 2.1 in the theory of queues is the following: consider a $G / M / 1$ queueing system with infinite capacity and deterministic, not necessarily equidistant arrival times $0<a_{1}<a_{2}<\cdots$. Assuming that the service times are exponential with mean $1 / \mu$, Stadje (1995) derived a determinant formula yielding the probability

$$
P\left(Q\left(a_{n}\right)=k, T_{m}>a_{n} \mid Q(0)=m\right),
$$

where $Q(t)$ is the number of customers in the system at time $t$ and

$$
T_{m}=\inf \{t: Q(t) \leq 0 \mid Q(0)=m\},
$$

the duration of a busy period initiated by $m>0$ customers. By Theorem 2.1 we can easily extend Stadje's formula to a queueing system where the service process is allowed to be time-inhomogeneous.

To see this, let us assume that the number of services $N(t)$ in the time interval $(0, t)$ is a nonstationary Poisson process with integrated intensity $\Lambda(t), \Lambda(0)=0$. Customers arrive at predetermined time instances $0<a_{1}<$ $a_{2}<\cdots$ and join the queue immediately after their arrivals at times $a_{i}+$. Since during a busy period the number of customers must be positive, the sequence $a_{n}$ gives rise to a moving boundary imposed on the nonstationary service process $N(t)$ counting the total number of completed services up to time $t$. The set of boundary points $\mathscr{C}_{n}$ is given by

$$
\mathscr{C}_{n}=\left\{\left(u_{i}, b_{i}\right), 1 \leq i \leq n\right\},
$$


with

$$
u_{i}=a_{i}, \quad b_{i}=m+i-1, \quad i=1,2, \ldots, n
$$

and

$$
u_{0}=b_{0}=0, \quad u_{n+1}=t, \quad b_{n+1}=m+n-k .
$$

These conditions follow from the fact that the event

$$
\left\{Q(t)=k, T_{m}>t\right\}, \quad a_{n}<t \leq a_{n+1}
$$

implies (i) the sample paths of $N(t)$ must not pass through any of the points in $\mathscr{C}_{n}$ and (ii) for the number of completed services up to time $t$ there must hold $N(t)=m+n-k$.

Thus

$$
P\left(Q(t)=k, T_{m}>t \mid Q(0)=m\right)=P_{n+1}, \quad a_{n}<t \leq a_{n+1},
$$

with $P_{0}=-1$ and

$$
\begin{aligned}
& P_{\ell}=-\sum_{i=0}^{\ell-1} \exp \left(-\left[\Lambda\left(u_{\ell}\right)-\Lambda\left(u_{i}\right)\right]\right) \frac{\left[\Lambda\left(u_{\ell}\right)-\Lambda\left(u_{i}\right)\right]^{b_{l}-b_{i}}}{\left(b_{l}-b_{i}\right) !} P_{i}, \\
& \quad \ell=1,2, \ldots, n+1 .
\end{aligned}
$$

Finally we give an application of Theorem 2.3.

Consider an $r$-node tandem queue and assume that the stream of customers entering the system at the first node is stationary Poisson with rate $\lambda_{0}>0$. Service times at the nodes are independent exponential random variables with mean $1 / \lambda_{i}$ at node $i=1, \ldots, r$. At time zero there are $m_{i}>0$ customers waiting at node $i$. Let $Q_{i}(t)$ denote the number of customers at node $i$ at time $t$. Define random stopping times

$$
T_{i}=\inf \left\{t: Q_{i}(t)=0\right\} .
$$

Also let $T=\min _{1 \leq i \leq r} T_{i}$.

The joint distribution of the random vector $\mathbf{Q}_{t}=\left(Q_{1}(t), \ldots, Q_{r}(t)\right)$ during a time period where all servers are continuously busy, that is, $T>t$, has been determined by Massey (1987) using an analytic approach based on lattice Bessel functions and by Böhm, Jain and Mohanty (1993) by means of lattice path counting in higher-dimensional spaces. Here we will demonstrate how their results may be obtained from Theorem 2.3.

Let

$$
p(\mathbf{m}, \mathbf{k}, t)=P\left(\mathbf{Q}_{t}=\mathbf{k}, T>t \mid \mathbf{Q}_{0}=\mathbf{m}\right),
$$

with

$\mathbf{k}=\left(k_{1}, k_{2}, \ldots, k_{r}\right), \quad \mathbf{m}=\left(m_{1}, m_{2}, \ldots, m_{r}\right), \quad k_{i}, m_{i}>0 \quad$ for $1 \leq i \leq r$.

Furthermore, let $n$ denote the total number of arrivals at node 1 during the time interval $(0, t)$. 
Now introduce Poisson counting processes $N_{0}(t), N_{1}(t), \ldots, N_{r}(t)$, where $N_{0}(t)$ counts the arrivals at node 1 and therefore has intensity $\lambda_{0}$, and the processes $N_{i}(t), 1 \leq i \leq r$, having intensities $\lambda_{i}$, count the service completions at the various nodes.

Since the output of node $i$ is the input of node $i+1$ and since we require that all nodes are continuously busy, the problem of finding $p(\mathbf{m}, \mathbf{k}, t)$ may be reduced to the determination of a noncoincidence probability of a set of dissimilar Poisson processes.

To see this let $N_{r}(t)$, the service process of the last node, start at height zero. At time zero there are $m_{r}$ customers already waiting at this node and furthermore the output process of node $r-1$ is the input process of node $r$. So let the counting process $N_{r-1}(t)$ start at height $m_{r}$. Since we require that the server at node $r$ is continuously busy throughout the time interval $(0, t)$, the processes $N_{r}(t)$ and $N_{r-1}(t)$ are not allowed to have a coincidence. Similarly let $N_{r-2}(t)$ start at height $m_{r}+m_{r-1}$, because there are $m_{r-1}$ customers initially waiting at node $r-1$. Again the processes $N_{r-2}(t)$ and $N_{r-1}(t)$ must be noncoincident.

More generally let $N_{i}(t)$ start at height $\sum_{\nu=i+1}^{r} m_{\nu}, 0 \leq i \leq r$ and interpret an empty sum as zero.

There are $n \geq 0$ arrivals at the first node in $(0, t)$. Therefore the process $N_{0}(t)$ terminates at height $n+\sum_{\nu=1}^{r} m_{\nu}$. The process $N_{1}(t)$ must terminate at height $n+\sum_{\nu=1}^{r} m_{\nu}-k_{1}$, since there are $k_{1}$ customers left at node 1 at time $t$. In general the processes $N_{i}(t)$ terminate at heights

$$
n+\sum_{\nu=1}^{r} m_{\nu}-\sum_{\nu=1}^{i} k_{\nu}
$$

Thus we have a set of $r+1$ independent Poisson processes starting at heights $\sum_{\nu=i+1}^{r} m_{\nu}$ and terminating at heights given by (24), which must be noncoincident.

Invoking Theorem 2.3, we get

$$
p(\mathbf{m}, \mathbf{k}, t)=\sum_{n \geq 0} \operatorname{det}\left\|\frac{1}{\left(b_{i}-a_{j}\right) !}\right\| \exp \left(-t \sum_{i=0}^{r} \lambda_{i}\right) \prod_{i=0}^{r}\left(\lambda_{i} t\right)^{b_{i}-a_{i}},
$$

with

$$
a_{i}=\sum_{\nu=i+1}^{r} m_{\nu}, \quad b_{i}=n+\sum_{\nu=1}^{r} m_{\nu}-\sum_{\nu=1}^{i} k_{\nu}, \quad i=0,1, \ldots, r .
$$

Acknowledgment. We greatly appreciate the constructive and helpful comments of the referee.

\section{REFERENCES}

BÖHM, W., JAIN, J. L. and MOHANTy, S. G. (1993). On zero-avoiding transition probabilities of an $r$-node tandem queue: a combinatorial approach. J. Appl. Probab. 30 737-741.

Fisher, M. E. (1984). Walks, walls, wetting and melting. J. Statist. Phys. 34 667-730. 
Gessel, I. and Viennot, G. (1985). Binomial determinants, paths and hook length formulae. Adv. in Math. 58 300-321.

KARLIN, S. (1988). Coincident probabilities and applications in combinatorics. J. Appl. Probab. 25A 185-200.

KarLin, S. and McGregor, J. G. (1957). The differential equations of birth-and-death processes and the Stieltjes moment problem. Trans. Amer. Math. Soc. 85 489-546.

Karlin, S. and McGregor, J. G. (1959). Coincidence probabilities. Pacific J. Math. 9 1141-1164.

Krattenthaler, C. and Mohanty, S. G. (1992). Counting tableaux with row and column bounds. In Proceedings of the 4th Colloque de Series Formelles et Combinatoire Algebraique, Montreal 1992 (C. Reutenauer and P. Leroux, eds.).

Massey, W. A. (1987). Calculating exit times for series Jackson networks. J. Appl. Probab. 27 226-234.

NoE, M. (1972). The calculation of two-sided Kolmogorov-Smirnov type statistics. Ann. Math. Statist. 43 58-64.

SHORACK, G. R. and WeLlner, J. A. (1986). Empirical Processes with Applications to Statistics. Wiley, New York.

SiEgmund, D. (1985). Sequential Analysis. Springer, New York.

STADJE, W. (1993). Distribution of first-exit times for empirical counting and Poisson processes with moving boundaries. Stochastic Models 9 91-103.

StadJE, W. (1995). The busy period of some queueing systems. Stochastic Process. Appl. 55 159167.

Stanley, R. P. (1986). Enumerative Combinatorics 1. Wadsworth \& Brooks, Belmont, CA.

ZACKS, S. (1991). Distributions of stopping times for Poisson processes with linear boundaries. Stochastic Models 7 233-242.

DEPARTMENT OF STATISTICS

UNIVERSITY OF ECONOMICS

A-1090 VIENNA

Augasse 2-6

AUSTRIA

E-MAIL: boehm@isis.wu-wien.ac.at
DEPARTMENT OF STATISTICS

MCMASTER UNIVERSITY

1280 MaIn StREet West

HAMILTON, ONTARIO

L8S4K1 CANADA

E-MAIL: mohanty@mcmaster.ca 